\title{
Increased expression of hypoxia-induced factor 1a mRNA and its related genes in myeloid blood cells from critically ill COVID-19 patients
}

\section{Keiko Taniguchi-Ponciano}

Unidad de Investigación Médica en Enfermedades Endocrinas, UMAE Hospital de Especialidades, Centro Medico Nacional Siglo XXI, Instituto Mexicano del Seguro Social.

\section{Eduardo Vadillo}

Unidad de Investigación Médica en Enfermedades Oncológicas, UMAE Hospital de Oncología, Instituto Mexicano del Seguro Social.

\section{Héctor Mayani}

Unidad de Investigación Médica en Enfermedades Oncológicas, UMAE Hospital de Oncología, Instituto Mexicano del Seguro Social.

\section{César Raúl Gonzales-Bonilla}

Titular, Coordinación de Investigación en Salud, Instituto Mexicano del Seguro Social.

\section{Javier Torres}

Unidad de Investigación Médica en Enfermedades Infecciosas y Parasitarias, UMAE Hospital de Pediatría, Instituto Mexicano del Seguro Social.

\section{Abraham Majluf}

Unidad de Investigación Médica en trombosis, hemostasia y aterogénesis, Instituto Mexicano del Seguro Social.

\section{Guillermo Flores-Padilla}

Servicio de Medicina Interna, UMAE Hospital de Especialidades, Centro Medico Nacional Siglo XXI, Instituto Mexicano del Seguro Social.

\section{Niels Wacher-Rodarte}

Unidad de Investigación Médica en Epidemiología Clínica, UMAE Hospital de Especialidades, Centro Medico Nacional Siglo XXI, Instituto Mexicano del Seguro Social.

\section{Juan Carlos Galan}

Servicio de Medicina Interna, UMAE Hospital de Especialidades, Centro Medico Nacional Siglo XXI, Instituto Mexicano del Seguro Social.

\section{Eduardo Ferat-Osorio}

División de Investigación en Salud, UMAE Hospital de Especialidades, Centro Medico Nacional Siglo XXI, Instituto Mexicano del Seguro Social.

\section{Francisco Blanco-Favela}


Unidad de Investigación Médica en Inmunología, UMAE Hospital de Pediatría, Centro Medico Nacional Siglo XXI, Instituto Mexicano del Seguro Social.

\section{Constantino Lopez-Macias}

Unidad de Investigación Médica en Inmunoquímica, UMAE Hospital de Especialidades, Centro Medico Nacional Siglo XXI, Instituto Mexicano del Seguro Social.

\section{Aldo Ferreira-Hermosillo}

Unidad de Investigación Médica en Enfermedades Endocrinas, UMAE Hospital de Especialidades, Centro Medico Nacional Siglo XXI, Instituto Mexicano del Seguro Social.

\section{Claudia Ramirez-Renteria}

Unidad de Investigación Médica en Enfermedades Endocrinas, UMAE Hospital de Especialidades, Centro Medico Nacional Siglo XXI, Instituto Mexicano del Seguro Social.

\section{Eduardo Peña-Martínez}

Unidad de Investigación Médica en Enfermedades Endocrinas, UMAE Hospital de Especialidades, Centro Medico Nacional Siglo XXI, Instituto Mexicano del Seguro Social.

\section{Gloria Silva-Román}

Unidad de Investigación Médica en Enfermedades Endocrinas, UMAE Hospital de Especialidades, Centro Medico Nacional Siglo XXI, Instituto Mexicano del Seguro Social.

\section{Sandra Vela-Patiño}

Unidad de Investigación Médica en Enfermedades Endocrinas, UMAE Hospital de Especialidades, Centro Medico Nacional Siglo XXI, Instituto Mexicano del Seguro Social.

\section{Carlos Mata-Lozano}

Unidad de Investigación Médica en Enfermedades Endocrinas, UMAE Hospital de Especialidades, Centro Medico Nacional Siglo XXI, Instituto Mexicano del Seguro Social.

\section{Roberto Carvente-Garcia}

Unidad de Investigación Médica en Enfermedades Endocrinas, UMAE Hospital de Especialidades, Centro Medico Nacional Siglo XXI, Instituto Mexicano del Seguro Social.

\section{Lourdes Basurto-Acevedo}

Unidad de Investigación Médica en Enfermedades Endocrinas, UMAE Hospital de Especialidades, Centro Medico Nacional Siglo XXI, Instituto Mexicano del Seguro Social.

\section{Renata Saucedo}

Unidad de Investigación Médica en Enfermedades Endocrinas, UMAE Hospital de Especialidades, Centro Medico Nacional Siglo XXI, Instituto Mexicano del Seguro Social.

\section{Patricia Piña-Sanchez}

Unidad de Investigación Médica en Enfermedades Oncológicas, UMAE Hospital de Oncología, Instituto Mexicano del Seguro Social.

\section{María Antonieta Chavez-Gonzalez}

Unidad de Investigación Médica en Enfermedades Oncológicas, UMAE Hospital de Oncología, Instituto Mexicano del Seguro Social.

Daniel Marrero-Rodríguez ( $\nabla$ dan.mar57@gmail.com ) 
Unidad de Investigación Médica en Enfermedades Endocrinas, UMAE Hospital de Especialidades, Centro Medico Nacional Siglo XXI, Instituto Mexicano del Seguro Social.

Moisés Mercado ( $\square$ moises.mercado@endocrinologia.org.mx )

Unidad de Investigación Médica en Enfermedades Endocrinas, UMAE Hospital de Especialidades, Centro Medico Nacional Siglo XXI, Instituto Mexicano del Seguro Social.

\section{Research Article}

Keywords: SARS-Cov-2, COVID-19, critically-ill, scRNAseq, HIF1A, myeloid

Posted Date: July 17th, 2020

DOl: https://doi.org/10.21203/rs.3.rs-43390/v1

License: (c) (1) This work is licensed under a Creative Commons Attribution 4.0 International License. Read Full License

Version of Record: A version of this preprint was published at Annals of Medicine on December 21st, 2020. See the published version at https://doi.org/10.1080/07853890.2020.1858234. 


\section{Abstract}

Since its emergence, in December 2019, COVID-19 has resulted in more than 12 million people infected and has killed more than 570000. Hypoxemia has been identified as one of the main clinical manifestations of this disease, especially in severe cases. We have previously reported that in critically ill COVID-19 patients there is a shift towards an immature myeloid profile in peripheral blood cells, including band neutrophils, immature monocytes, metamyelocytes, monocyte-macrophages, monocytoid precursors, and promyelocytes-myelocytes, which, together with mature monocytes and segmented neutrophils, comprise the vast majority of blood cells in these patients. Such an immature myeloid profile may be the result of a physiological response known as emergency myelopoiesis. In the present study, we performed scRNAseq from leukocytes from five critically ill COVID-19 patients and characterized the expression of hypoxia-inducible factor1a (HIF1a) mRNA and its transcriptionally regulated genes. HIF1a is a master transcription factor involved in the cellular response to hypoxia. We herein report that these cellular subsets express high levels of HIF1a mRNA and several of their transcriptional targets, including those related to inflammation, such as CXCL8, CXCR1, CXCR2, and CXCR4; those potentially involved in virus sensing, such as TLR2 and TLR4; and those related to metabolism, such as SLC2A3, PFKFB3, PGK1, GAPDH and SOD2. The up-regulation and participation of HIF1a in relevant events such as inflammation, immunometabolism, and TLR make it a potential molecular marker for COVID-19 severity and, interestingly, could represent a potential target for molecular therapy.

\section{Introduction}

The coronavirus disease 2019 (COVID-19) epidemic caused by the severe acute respiratory syndrome coronavirus $2^{1}$ has rapidly developed into a devastating pandemic ${ }^{1}$. As of today, the World Health Organization has reported more than 12 million persons diagnosed with COVID-19 and over 570000 deaths worldwide ${ }^{2}$. It is associated with significant mortality in high risk patients, with poor prognostic features upon admission. The spectrum of the disease is broad, including pneumonia, sepsis, and acute respiratory distress syndrome (ARDS) ${ }^{3}$. Hypoxemia, defined as a decrease in the partial pressure of oxygen is an ominous sign of COVID-19, and it is usually an indicator of disease severity 4,5 . An oxygen saturation above $90 \%$ is associated with better outcomes ${ }^{6}$. Over $80 \%$ of COVID-19 patients in the intensive care unit have severe hypoxemia ${ }^{7}$. A kind of "silent hypoxia" in which COVID-19 patients deteriorate rapidly without warning and develop respiratory failure has been described ${ }^{8}$. Hypoxia indicates an imbalance of oxygen delivery to tissues and leads to compromised function, which is quantitatively related to organ, tissue and even cell type ${ }^{9}$. The hypoxia-inducible factors (HIF) are considered master regulators of oxygen homeostasis and are oxygen level sensitive ${ }^{10}$. Currently, there is scarce information regarding the expression of HIF in patients with severe COVID-19 and its potential involvement in the immunopathogenesis of this condition. Therefore, in the present work we carried out scRNAseq to identify the cell populations present in critically ill COVID-19 patients and to determine the expression of hypoxia-induced factor $1 \mathrm{a}(\mathrm{HIF} 1 \mathrm{a})$ and its related genes. 


\section{Materials And Methods \\ Patients and tissue samples}

Blood samples from five critically ill patients with COVID-19 were collected in EDTA-coated tubes.

Tissues were collected from patients diagnosed, treated and followed at the Medicina Interna department of the Hospital de Especialidades, Centro Médico Nacional Siglo XXI of the Instituto Mexicano del Seguro Social in April 2020. A family member of each participating patient signed an informed consent and the study protocol was approved by the Comisión Nacional de Ética e Investigación Científica del Instituto Mexicano del Seguro Social in accordance to the Helsinki declaration. SARS-CoV-2 infection was corroborated by RT-qPCR at an official federal government reference laboratory.

\section{Sample preparation, scRNAseq library generation and sequencing}

Peripheral blood from the five critical COVID-19 patients was collected in EDTA-coated tubes, and immune cells were isolated according to standard centrifugation methods followed by red blood cell lysis.

Chromium Next GEM Single Cell 3' Reagent Kits v3.1 and protocol from 10X Genomics was followed as recommended by manufacturer's instructions. Briefly, immune cells were pooled in a single tube and cells were diluted in $1 x$ phosphate buffered saline (PBS) to 700-1200 cells per $\mu$ l. Cell suspension was loaded in Chromium Next GEM Chip G and sorted in the Chromium Controller from 10X Genomics. The Cell-Gel Beads in Emulsion (GEMs) were then incubated to generate the barcoded cDNA. cDNA was cleaned using Dynabeads and washed, followed by cDNA amplification and SPRIselection. The retrieved cDNA was enzymatically fragmented, end-repaired, poly-A tailed and ligated. Size selection, adaptor ligation and amplification were done. Sequencing was done using NextSeq 550 System High-Output Kit (300 cycles) in NextSeq 500 system (Illumina) according to 10X Genomics specifications: Read $1=28$ cycles, Read 2 = 91 cycles, Index 1 = 8 cycles. All quality control steps were carried out using 4200 TapeStation System (Agilent) with High Sensitivity D1000 Screen Tape, whereas the concentration was calculated using Qubit 2.0 Fluorometer with Kit High Sensitivity assays.

\section{scRNAseq bioinformatic analysis}

Partek Flow software was used with scRNAseq toolbox. First the tags were trimmed and then the reads were aligned using STAR 2.7.3a algorithm to human genome hg38. UMl's were deduplicated and barcode filtered. Following criteria were then applied to each cell, i.e., gene number between 200 and $6000, \mathrm{UMI}$ count above 300 and mitochondrial gene percentage below $20 \%$. To quantify the transcriptome human hg38 Ensembl transcripts release 99 was used. Counts per million, Add 1.0 Log 2.0 were the normalization parameters. Healthy donors' datasets were downloaded from 10X Genomics website and 


\section{Markers used to circumscribe cell populations}

Clusters were categorized by analyzing differentially expressed genes according to previously published data obtained from human samples ${ }^{11-16}$

\section{Dimensionality reduction and clustering}

The filtered and normalized gene-barcode matrix was analyzed by principal components, then graph based and t-distributed stochastic neighbor embedding (t-SNE) using default parameters was carried out.

\section{Results}

\section{Immature myeloid cell populations in critically ill COVID-19 patients}

We have previously reported that, as compared to healthy adults, lymphoid cell subsets, such as B and T lymphocytes as well as NK cells, were present in low quantities in critically ill COVID-19 patients, whereas cells of myeloid origin predominated. Interestingly, immature myeloid cell populations, such as band neutrophils, metamyelocytes, promyelocytes-myelocytes, monocytoid precursor, and immature monocytes prevailed. Mature lineages such as segmented neutrophils, mature monocytes and finally monocyte-macrophages were also observed (Figure 1) ${ }^{17}$.

\section{HIF1a expression in leukocytes from critically ill COVID-19 patients}

Once the blood cell populations were identified, we looked for HIF1a gene expression. As shown in Figure 2A, HIF1a gene was expressed in all myeloid lineages to a greater extent than in lymphoid cells. This was particularly evident in the mature monocyte population. Since HIF1a is a transcription factor that acts as a trans regulator, we searched for HIF1a-regulated genes potentially involved in COVID-19 immunity. Among these immune related genes, we found an increased expression of CXCL8 or Interleukin-8, a chemokine involved in the migration of mature neutrophils to the site of infection in most myeloid cell subsets, and almost no expression in lymphoid populations and monocytoid precursors (Figure 2B). In keeping with the increased expression of CXCL8, the genes for chemokine receptors CXCR2 (Figure 2C) and CXCR4 (Figure 2D), and also CXCR1 were also expressed at increased levels in most myeloid lineages. It is noteworthy that lymphoid cells did express the CXCR4 gene, but showed no expression of the CXCR2 gene, which can explain the exacerbated inflammatory response characteristic of these 
patients ${ }^{18}$. Interestingly, we found expression of Toll like receptor-2 and -4 (TLR2 and TLR4) in most myeloid populations, which could be related to SARS-CoV-2 sensing (Figure 2E and 2F).

Along with the identified genes regulated by HIF1a, we found expression of genes related to metabolism such as solute carrier family 2 member 3 (SLC2A3) also known as GLUT3, 6-phosphofructo-2-kinase (PFKFB3), phosphoglycerate kinase 1 (PGK1) and glyceraldehyde-3-P-dehydrogenase (GAPDH) (Figure 3). This latter related with the neutrophil survival through the inhibition of their programed cell death ${ }^{19}$.

We also found expression of superoxide dismutase 2 (SOD2), which is involved in the metabolism of reactive oxygen species ${ }^{20}$, vimentin (VIM) a type III intermediate filament and plasminogen activator urokinase receptor (PLAUR), which is related to plasminogen activation.

Considering that HIF1 a function is controlled by different factors, we also evaluated the expression of the Von Hippel Lindau (VHL) and prolyl-4-hydroxylase (P4HA1) genes, two of the main inhibitors of HIF1a function. Indeed, these two molecules are involved in the ubiquitination and degradation of HIF1a. In keeping with the increased expression of HIF1a and target genes observed, we found that neither VHL nor P4HA1 were expressed by the peripheral blood cells of critically ill COVID-19 patients (Figure $2 \mathrm{G}$ and $2 \mathrm{H}$ ).

We next evaluated the potential interaction between HIF1a and their transcriptional targets by assessing the simultaneous presence of their mRNAs in the same single cells (Figure 4). As shown in Figure 4A, a significant proportion of myeloid cells co-expressed HIF1a and CXCL8. We also observed a predominance of cells co-expressing HIF1a and TLR2 (Figure 4B) as well as HIF1a and SOD2 (Figure 4C). It is noteworthy that among the different myeloid subsets, mature monocytes were the ones that exhibiting co-expression of most of the analyzed genes.

Finally, we evaluated the expression of HIF1a and its transcriptional targets in peripheral blood cells from healthy donors. To do so, we analyzed $10 \mathrm{X}$ Genomics publicly available datasets. We observed that HIF1a expression was lower as compared to the expression observed in COVID-19 patients (Figure 5). Similar results were observed for genes such as CXCL8, CXCR2, PLAUR, TLR4 and SOD2 (Figure 5).

\section{Discussion}

Hypoxia and inflammation are unequivocally linked ${ }^{21}$ and are two of the main physiological consequences of SARS-CoV-2 infection, particularly in severe cases. In this study we present scRNAseq data regarding HIF1a-related gene expression in peripheral blood leukocytes from critically ill COVID-19 and characterized the different cell subpopulations. HIF1a is a heterodimeric transcription factor sensitive to oxygen and induced under hypoxic conditions ${ }^{22}$. The HIF1a trans element can regulate the expression of CXCL8 ${ }^{23}$, CXCR1, CXCR2 ${ }^{24}$ and CXCR4 ${ }^{25}$. CXCL8 expression can be stimulated by interleukin (IL) 6 , TNFa, hypoxia ${ }^{26}$ and viral infection ${ }^{27}$ in cells such as monocytes, neutrophils, epithelial cells and fibroblasts ${ }^{28}$. CXCL8 is a chemokine that exerts its pro-inflammatory functions throughout the CXCR1 and CXCR2 receptors. CXCL8 and its receptors contribute to pathogen elimination, through the transient 
activation of ERK, AKT, SRC and FAK leading to activation of neutrophils ${ }^{26}$. The expression of CXCL8, which is present in COVID-19 patients, is considered a potential prognostic factor in acute respiratory distress syndrome (ARDS) ${ }^{29}$ and lung injury ${ }^{30}$.

The SARS-CoV-2 viral entry depends upon binding of viral spike (S) protein to the host cell surface protein angiotensin-converting enzyme 2 (ACE2) ${ }^{20}$. The immunopathological outcomes are most likely induced by the host-virus interaction. The interaction between viral antigen and host immune cells results in an exacerbated inflammatory response ${ }^{31}$. In the present study, we also found high expression of both TLR2 and TLR4 genes in peripheral blood leukocytes of severe COVID-19 cases. The viral Spike protein can be recognized by TLR2 ${ }^{32}$ and TLR4 ${ }^{31}$, which are up-regulated in the presence of another coronavirus such as SARS-CoV ${ }^{33}$. TLR4 constitutes one of the most efficient innate immune receptors, triggering proinflammatory responses after binding to the pathogenic ligand, and this interaction could be useful for developing drugs ${ }^{31,34}$.

Metabolic reprograming of innate immune cells occurs during hyperinflammatory states. Immune cells contribute to systemic changes in metabolism by altering their metabolic profiles in response to the immunological state. Therefore, therapeutic modulation of immune cell metabolism could alter the inflammatory state and thus improve patient prognosis ${ }^{35}$. Inflammation and hypoxia are inherently linked, and hypoxia is a well-known glycolysis driver as oxygen deficit results in limited OXPHOS ${ }^{36}$. Previous studies have shown that the molecular mechanisms underlying the switch from OXPHOS to glycolysis during innate immune cell response require HIF1a ${ }^{35}$. Among the HIF1a responsive genes, we found expression of those related to carbohydrate metabolism, such as SLC2A3/GLUT3, PFKFB3, PGK1 and GAPDH ${ }^{37}$. HIF signaling pathway activation in neutrophils results in an increased survival of these cells, $\beta 2$ integrin expression, production of antimicrobial peptides and glycolysis. Neutrophils use high rates of Warburg-like glycolysis for ATP generation. The Absence of HIF1a leads to reduced ATP pools resulting in a profound impairment of the inflammatory response ${ }^{38}$. HIF1 a can also regulate nitric oxide production, pentose phosphate pathway, OXPHOS and arginase metabolism ${ }^{35}$. Overall, immunometabolism is now considered an indispensable regulator of immunity, with HIF1a playing a central role, modulating the function of various immune cell subsets ${ }^{38}$. The expression of HIF1a has been previously found to be a sepsis marker ${ }^{35}$.

HIF1 a participates in the regulation of a plethora of cellular events such as metabolism of ROS trough the regulation of SOD2 ${ }^{39}$, the regulation of cytoskeleton trough VIM type III filament, which also participates in inflammation ${ }^{40}$, and PLAUR which activates plasminogen and activates a cascade of extracellular proteases ${ }^{41}$. Interestingly, the expression of this gene could be used as a predictor of severe respiratory failure ${ }^{42}$ which is consistent with our results.

In conclusion, in the present study, we have demonstrated the expression of HIF1a and its transcriptionally regulated genes, in myeloid cells, including both mature and immature subsets, present in peripheral blood of critically ill COVID-19 patients. The up-regulation and participation of HIF1a in 
relevant events such as inflammation, immunometabolism, and TLR supports its use as molecular marker for COVID-19 severity and as a potential candidate for targeted therapy.

\section{Declarations}

\section{Acknowledgments}

DMR is a recipient of the National Council for Science and Technology Fellowship "Catedra CONACyT" program. This work was partially supported by grants 289499 from Fondos Sectoriales Consejo Nacional de Ciencia y Tecnologia Mexico and R-2015-785-015 from Instituto Mexicano del Seguro Social (MM). We would like to thank to Xiaowen Wang from Partek Inc. for the exceptional technical support provided.

\section{Author contributions}

MM, DMR and KTP conceived, designed and coordinated the project, performed scRNAseq experiments, analyzed, discussed data and prepared the manuscript. EV, HM, CRGB, JT, AM, NWR, FBF, AFH, CRR, EPM, GSR, SVP, CML, RCG, LBA, RS, PPS and ACG performed scRNAseq experiments, analyzed, discussed and interpreted biological data, wrote the paper.

CLM, JCG, EFO, GFP, provided the biological sample, retrieved the immune cells and collect clinical data.

\section{Competing interest}

RCG and CML work for Analitek S.A. de C.V. which supplied research reagents. The rest of the authors declare not competing interests.

\section{References}

1. Bi Q, Wu Y, Mei S, et al. Epidemiology and transmission of COVID-19 in 391 cases and 1286 of their close contacts in Shenzhen, China: a retrospective cohort study. Lancet Infect Dis. 2020.

2. Coronavirus disease (COVID-2019) situation reports 176. 2020.

3. Jose RJ, Manuel A. COVID-19 cytokine storm: the interplay between inflammation and coagulation. Lancet Respir Med. 2020;8(6):e46-e47.

4. Sarkar M, Niranjan N, Banyal PK. Mechanisms of hypoxemia. Lung India. 2017;34(1):47-60.

5. Kashani KB. Hypoxia in COVID-19: Sign of Severity or Cause for Poor Outcomes. Mayo Clin Proc. 2020;95(6):1094-1096.

6. Xie J, Covassin N, Fan Z, et al. Association Between Hypoxemia and Mortality in Patients With COVID-19. Mayo Clin Proc. 2020;95(6):1138-1147.

7. Cavezzi A, Troiani E, Corrao S. COVID-19: hemoglobin, iron, and hypoxia beyond inflammation. A narrative review. Clin Pract. 2020;10(2):1271. 
8. Ottestad W, Seim M, Maehlen JO. COVID-19 with silent hypoxemia. Tidsskr Nor Laegeforen. 2020;140(7).

9. Choudhury R. Hypoxia and hyperbaric oxygen therapy: a review. Int J Gen Med. 2018;11:431-442.

10. Majmundar AJ, Wong WJ, Simon MC. Hypoxia-inducible factors and the response to hypoxic stress. Mol Cell. 2010;40(2):294-309.

11. Hoogendijk AJ, Pourfarzad F, Aarts CEM, et al. Dynamic Transcriptome-Proteome Correlation Networks Reveal Human Myeloid Differentiation and Neutrophil-Specific Programming. Cell Rep. 2019;29(8):2505-2519 e2504.

12. Grassi L, Pourfarzad F, Ullrich S, et al. Dynamics of Transcription Regulation in Human Bone Marrow Myeloid Differentiation to Mature Blood Neutrophils. Cell Rep. 2018;24(10):2784-2794.

13. Novershtern N, Subramanian A, Lawton LN, et al. Densely interconnected transcriptional circuits control cell states in human hematopoiesis. Cell. 2011;144(2):296-309.

14. Lambert C, Preijers F, Yanikkaya Demirel G, Sack U. Monocytes and macrophages in flow: an ESCCA initiative on advanced analyses of monocyte lineage using flow cytometry. Cytometry B Clin Cytom. 2017;92(3):180-188.

15. Itelman E, Wasserstrum Y, Segev A, et al. Clinical Characterization of 162 COVID-19 patients in Israel: Preliminary Report from a Large Tertiary Center. Isr Med Assoc J. 2020;22(5):271-274.

16. Tang-Huau TL, Gueguen P, Goudot C, et al. Human in vivo-generated monocyte-derived dendritic cells and macrophages cross-present antigens through a vacuolar pathway. Nat Commun. 2018;9(1):2570.

17. Taniguchi-Ponciano K, Vadillo E, Lopez-Macias C, et al. A shift towards an immature myeloid profile in peripheral blood of critically ill COVID-19 patients. Research Square. 2020:1-20.

18. Sadiku P, Walmsley SR. Hypoxia and the regulation of myeloid cell metabolic imprinting: consequences for the inflammatory response. EMBO Rep. 2019;20(5).

19. Walmsley SR, Print C, Farahi N, et al. Hypoxia-induced neutrophil survival is mediated by HIF-1alphadependent NF-kappaB activity. J Exp Med. 2005;201(1):105-115.

20. Hoffmann M, Kleine-Weber H, Schroeder S, et al. SARS-CoV-2 Cell Entry Depends on ACE2 and TMPRSS2 and Is Blocked by a Clinically Proven Protease Inhibitor. Cell. 2020;181(2):271-280 e278.

21. Vanderhaeghen $\mathrm{T}$, Vandewalle J, Libert C. Hypoxia-inducible factors in metabolic reprogramming during sepsis. FEBS J. 2020;287(8):1478-1495.

22. Masoud GN, Li W. HIF-1alpha pathway: role, regulation and intervention for cancer therapy. Acta Pharm Sin B. 2015;5(5):378-389.

23. Imtiyaz HZ, Simon MC. Hypoxia-inducible factors as essential regulators of inflammation. Curr Top Microbiol Immunol. 2010;345:105-120.

24. Maxwell PJ, Gallagher R, Seaton A, et al. HIF-1 and NF-kappaB-mediated upregulation of CXCR1 and CXCR2 expression promotes cell survival in hypoxic prostate cancer cells. Oncogene. 2007;26(52):7333-7345. 
25. Ishikawa T, Nakashiro K, Klosek SK, et al. Hypoxia enhances CXCR4 expression by activating HIF-1 in oral squamous cell carcinoma. Oncol Rep. 2009;21(3):707-712.

26. Ha H, Debnath B, Neamati N. Role of the CXCL8-CXCR1/2 Axis in Cancer and Inflammatory Diseases. Theranostics. 2017;7(6):1543-1588.

27. Tang FS, Van Ly D, Spann K, et al. Differential neutrophil activation in viral infections: Enhanced TLR7/8-mediated CXCL8 release in asthma. Respirology. 2016;21(1):172-179.

28. Qazi BS, Tang K, Qazi A. Recent advances in underlying pathologies provide insight into interleukin-8 expression-mediated inflammation and angiogenesis. Int J Inflam. 2011;2011:908468.

29. Coperchini F, Chiovato L, Croce L, Magri F, Rotondi M. The cytokine storm in COVID-19: An overview of the involvement of the chemokine/chemokine-receptor system. Cytokine Growth Factor Rev. 2020;53:25-32.

30. Russo RC, Garcia CC, Teixeira MM, Amaral FA. The CXCL8/IL-8 chemokine family and its receptors in inflammatory diseases. Expert Rev Clin Immunol. 2014;10(5):593-619.

31. Choudhury A, Mukherjee S. In silico studies on the comparative characterization of the interactions of SARS-CoV-2 spike glycoprotein with ACE-2 receptor homologs and human TLRs. J Med Virol. 2020.

32. Dosch SF, Mahajan SD, Collins AR. SARS coronavirus spike protein-induced innate immune response occurs via activation of the NF-kappaB pathway in human monocyte macrophages in vitro. Virus Res. 2009;142(1-2):19-27.

33. Hu W, Yen YT, Singh S, Kao CL, Wu-Hsieh BA. SARS-CoV regulates immune function-related gene expression in human monocytic cells. Viral Immunol. 2012;25(4):277-288.

34. Choudhury A, Mukherjee S. In silico studies on the comparative characterization of the interactions of SARS-CoV-2 spike glycoprotein with ACE-2 receptor homologs and human TLRs. Journal of medical virology. 2020:10.1002/jmv.25987.

35. Fitzpatrick SF. Immunometabolism and Sepsis: A Role for HIF? Front Mol Biosci. 2019;6:85.

36. Corcoran SE, O'Neill LA. HIF1alpha and metabolic reprogramming in inflammation. J Clin Invest. 2016;126(10):3699-3707.

37. Liu W, Shen SM, Zhao XY, Chen GQ. Targeted genes and interacting proteins of hypoxia inducible factor-1. Int J Biochem Mol Biol. 2012;3(2):165-178.

38. Krzywinska E, Stockmann C. Hypoxia, Metabolism and Immune Cell Function. Biomedicines. 2018;6(2).

39. Gao YH, Li CX, Shen SM, et al. Hypoxia-inducible factor 1alpha mediates the down-regulation of superoxide dismutase 2 in von Hippel-Lindau deficient renal clear cell carcinoma. Biochem Biophys Res Commun. 2013;435(1):46-51.

40. Su L, Pan P, Yan P, et al. Role of vimentin in modulating immune cell apoptosis and inflammatory responses in sepsis. Sci Rep. 2019;9(1):5747. 
41. Gilder AS, Natali L, Van Dyk DM, et al. The Urokinase Receptor Induces a Mesenchymal Gene Expression Signature in Glioblastoma Cells and Promotes Tumor Cell Survival in Neurospheres. Sci Rep. 2018;8(1):2982.

42. Rovina N, Akinosoglou K, Eugen-Olsen J, Hayek S, Reiser J, Giamarellos-Bourboulis EJ. Soluble urokinase plasminogen activator receptor (suPAR) as an early predictor of severe respiratory failure in patients with COVID-19 pneumonia. Crit Care. 2020;24(1):187.

\section{Figures}



Figure 1

t-distributed stochastic neighbor embedding map (t-SNE) showing the identification of 12 cell clusters in critically ill COVID-19 patients. 


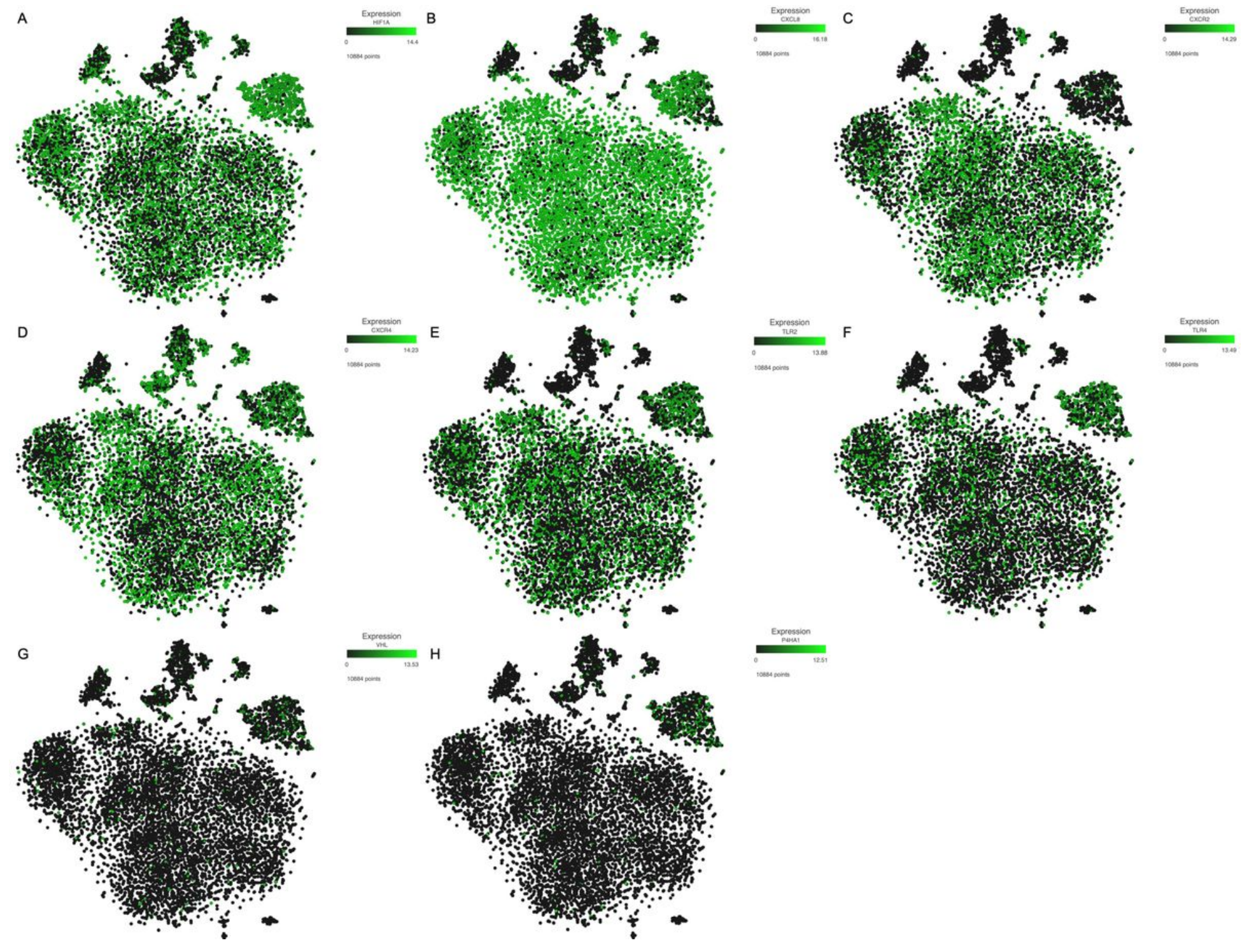

Figure 2

Expression levels of HIF1a and transcriptionally regulated target genes in peripheral blood cell lineages.



Figure 3

Violin plots from HIF1a transcriptionally regulated genes. 


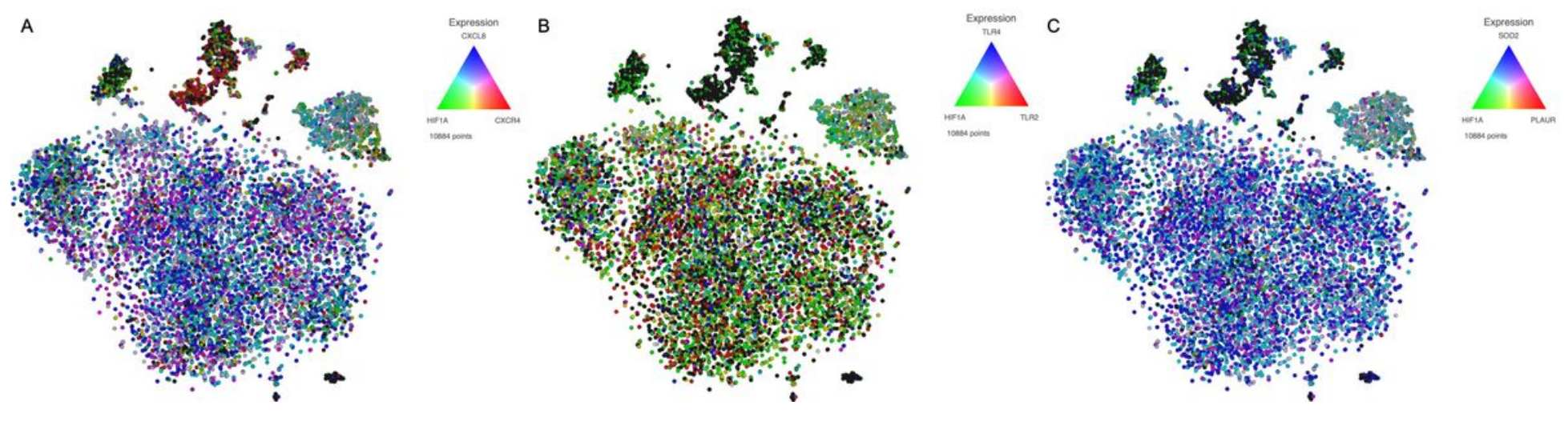

Figure 4

Simultaneous expression of HIF1a and target genes on the same single cell.

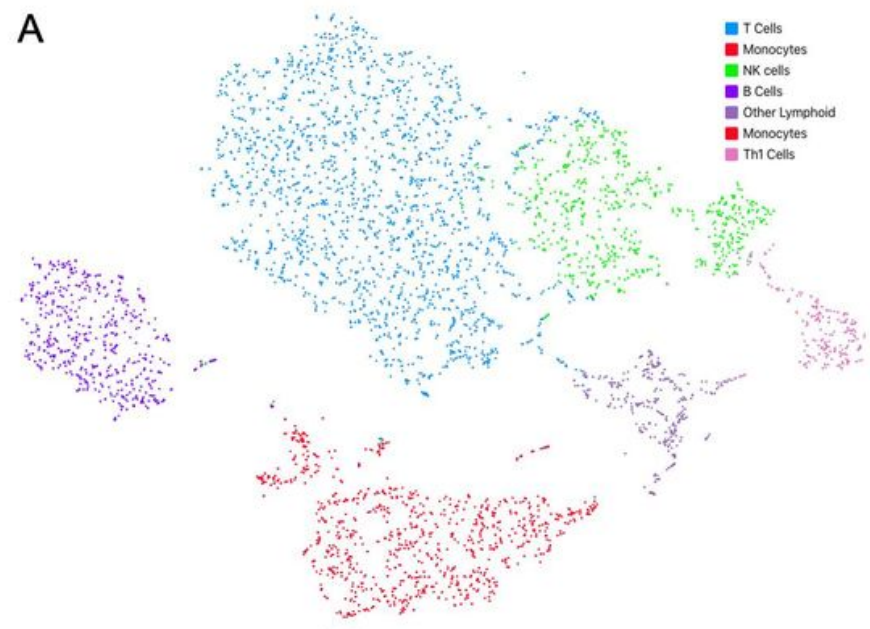

B

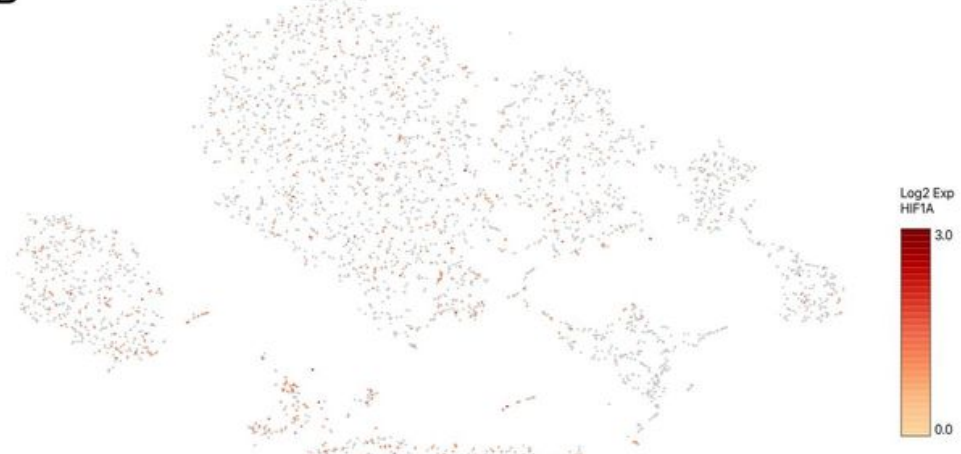

C
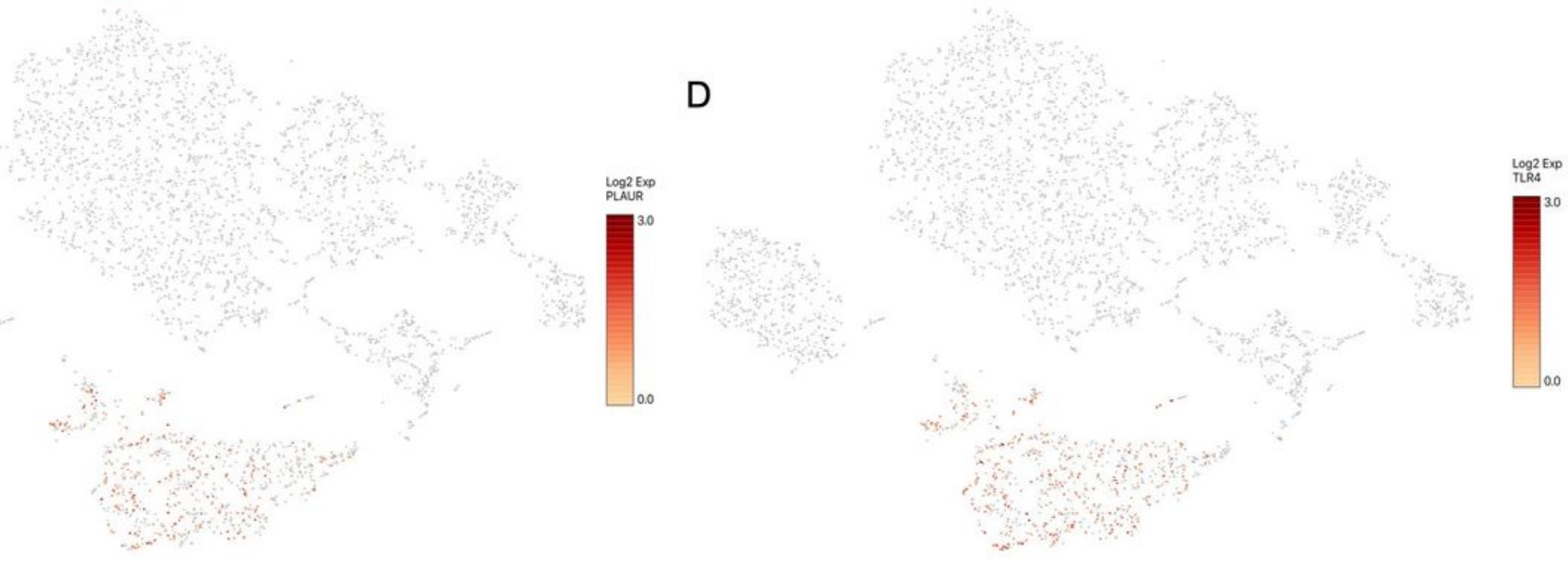

Figure 5

Cell populations and HIF1a gene expression identified in peripheral blood cells from healthy individuals. 